INPLASY

PROTOCOL

To cite: Wang et al. Treatment of vertebral artery type cervical spondylosis with Traditional Chinese medicine. Inplasy protocol 2021120004. doi: 10.37766/inplasy2021.12.0004

Received: 01 December 2021

Published: 01 December 2021

Corresponding author: Tenglong Li

2005225@huucm.edu.cn

Author Affiliation:

The Second Affiliated Hospital

of Hunan University of

Chinese MedicineHunan

University of Chinese

Medicine.

\section{Support: Project of Education} Department.

Review Stage at time of this submission: Formal screening of search results against eligibility criteria.

Conflicts of interest:

None declared.

\section{Treatment of vertebral artery type cervical spondylosis with Traditional Chinese medicine}

\author{
Wang, YX1'; Li, TL2; Xiong, H³; Li, L4; Yang, L5; Shu, Y6.
}

Review question / Objective: Participants: vertebral artery type of cervical spondylosis; Intervention: Oral Chinese medicine or proprietary Chinese medicine treatment; Control: Oral treatment of western medicine; Outcome:Total effective rate, grade evaluation of cure rate, transcranial Doppler detection of basilar hemodynamics index; Study design: randomized controlled trial.

Condition being studied: At present, oral western medicine treatment of vertebral artery type cervical spondylosis is not effective, and there are side effects, reducing the quality of life of patients. Traditional Chinese medicine or proprietary Chinese medicine has a significant therapeutic effect on vertebral artery cervical spondylosis, which has been recognized by most clinicians and patients in China. In order to further clarify the therapeutic effect of traditional Chinese medicine on vertebral artery cervical spondylosis and provide evidence for clinical practice, we designed this study.

INPLASY registration number: This protocol was registered with the International Platform of Registered Systematic Review and Meta-Analysis Protocols (INPLASY) on 01 December 2021 and was last updated on 04 December 2021 (registration number INPLASY2021120004).

\section{INTRODUCTION}

Review question / Objective: Participants: vertebral artery type of cervical spondylosis; Intervention: Oral Chinese medicine or proprietary Chinese medicine treatment; Control: Oral treatment of western medicine; Outcome:Total effective rate, grade evaluation of cure rate, transcranial Doppler detection of basilar hemodynamics index; Study design: randomized controlled trial.

Condition being studied: At present, oral western medicine treatment of vertebral 
artery type cervical spondylosis is not effective, and there are side effects, reducing the quality of life of patients. Traditional Chinese medicine or proprietary Chinese medicine has a significant therapeutic effect on vertebral artery cervical spondylosis, which has been recognized by most clinicians and patients in China. In order to further clarify the therapeutic effect of traditional Chinese medicine on vertebral artery cervical spondylosis and provide evidence for clinical practice, we designed this study.

\section{METHODS}

Search strategy: Databases were searched, including Chinese National Knowledge Infrastructure (CNKI), wanfang database, Wiper database and Chinese Biomedical Database. English databases include PubMed, Embase, Cochrane Library, and Web of Science. The search period was From January 2011 to November 2021. According to the different retrieval methods of database, Boolean logic operation or keyword and free word retrieval method is used for retrieval. Chinese search terms included "vertebral artery type cervical spondylosis", "cervical spondylosis", "Traditional Chinese medicine", "Proprietary Chinese medicine", "randomized control", etc. English search words included "cervical spondylotic arteriopathy", "Traditional Chinese medicine", "Chinese patent medicine", "randomized" Controlled trials ", etc. Multiple searches were made to ensure that the literature was as complete as possible. Contact experts and researchers in related fields to ensure that we can keep abreast of relevant research progress in the industry.

Participant or population: For patients clinically diagnosed with vertebral artery type cervical spondylosis, the diagnostic criteria should be recognized standards, excluding other cardiovascular and cerebrovascular diseases. There is no restriction on the course of treatment, nationality or country.
Intervention: Oral Chinese medicine or proprietary Chinese medicine treatment.

Comparator: Oral Western medicine.

Study designs to be included: All randomized controlled trials (RCTS) of TCM (single drug, group prescription) or proprietary Chinese medicine (including oral and external application) in the treatment of CSA have been published and can be found in various databases, regardless of language or race.

Eligibility criteria: Any of the following conditions will be excluded :(1) duplicate literature; (2) Expert experience summary, review literature, Meta analysis and systematic review; (3) Non-clinical RCT, unable to obtain full-text literature; (4) The treatment group does not include Traditional Chinese medicine or proprietary Chinese medicine, or the treatment group has Western medicine, or the treatment group contains auxiliary measures not used in the control group, or the control group includes traditional Chinese medicine and proprietary Chinese medicine; (5) Too many auxiliary intervention measures (more than one); (6) Outcome indicators cannot be counted; (7) The total number of subjects was less than 30 ; (8) The literature quality is too low.

Information sources: Databases were searched, including Chinese National Knowledge Infrastructure (CNKI), wanfang database, Wiper database and Chinese Biomedical Database. English databases include PubMed, Embase, Cochrane Library, and Web of Science. When the full text cannot be obtained or the data is insufficient, you can contact the author of the article to obtain specific data.

Main outcome(s): The total response rate was the primary outcome index, and the secondary outcome indexes included other grade data effective indexes, visual pain scale (VAS), TCD blood vessel evaluation, and the occurrence of adverse reactions. 
Quality assessment / Risk of bias analysis: The Cochrane Systematic Review Manual and the Jadad Scale.

Strategy of data synthesis: Review Manager 5.3 and Stata 16.

Subgroup analysis: Subgroup analysis can be performed if heterogeneity exists in the included study and heterogeneity can be found.

Sensitivity analysis: Sensitivity analysis was performed when heterogeneity existed in the included studies and the source of heterogeneity needed to be found.

Language: Not subject to any language restrictions.

Country(ies) involved: China.

Keywords: Traditional Chinese medicine; Chinese patent medicine; Vertebral artery type cervical spondylosis; Cervical spondylosis.

Contributions of each author:

Author 1 - Wang Yixuan completed the overall work of research design, organization and article writing, Li Tenglong and Xiong Hui provided technical guidance and organization coordination, Shu Yang and $\mathrm{Li} \mathrm{Lu}$ conducted literature screening and data collection, Wu Boyu and Yang Lei mainly completed literature evaluation, and Zeng Peng participated in arbitration.

Email: yx.wang.work@outlook.com

Author 2 - Tenglong Li.

Email: 2005225@huucm.edu.cn

Author 3 - Xiong Hui provided technical guidance and organization coordination.

Author 4 - $\mathrm{Li}$ Lu conducted literature screening and data collection.

Email: 864855166@qq.com

Author 5 - Lei Yang.

Author 6 - Yang Shu. 